\title{
Postmastectomy Scar Boost Irradiation Using HDR Surface Mould Brachytherapy by 3D Image-Based Volume Optimization
}

\author{
Neelakandan Vijayaprabhu ${ }^{1}$, Karunanithi Gunaseelan ${ }^{1 *}$, Nagarajan Vivekanandan ${ }^{2}$, \\ Nagamuthu Karthik ${ }^{3}$, Cholayil Shamsudheen ${ }^{1}$, K. S. Reddy ${ }^{1}$ \\ ${ }^{1}$ Department of Radiotherapy, Regional Cancer Center, Jawharlal Institute of Postgraduate Medical Education \\ and Research (JIPMER), Puducherry, India \\ ${ }^{2}$ Department of Medical Physics, Cancer Institute (WIA), Chennai, India \\ ${ }^{3}$ Department of Medical Physics, Anna University, Chennai, India \\ Email: vijayaprabhu.n@gmail.com,*gunapgi@gmail.com, viveknaren@hotmail.com, carthic0301@gmail.com, \\ shamsu48@gmail.com, atsahara11@yahoo.com
}

Received September 19, 2013; revised October 20, 2013; accepted November 2, 2013

Copyright (C) 2013 Neelakandan Vijayaprabhu et al. This is an open access article distributed under the Creative Commons Attribution License, which permits unrestricted use, distribution, and reproduction in any medium, provided the original work is properly cited.

\begin{abstract}
Introduction: During postmastectomy radiotherapy (PMRT), it is recommended to boost the postmastectomy surgical scar with additional $10 \mathrm{~Gy}$ in 5 fractions in the patients with close or positive surgical margins. The electron beam therapy, though cumbersome, is usually preferred since it has the desired rapid fall of a dose beyond $\mathrm{R}_{85}$. An alternative but easier and reproducible treatment method for PMRT surgical scar boost using 3D CT image-based HDR surface mould brachytherapy is introduced and analyses of the target coverage and dose nearby organs-at-risk (OARs) using this method are evaluated in this study. Methods and Materials: This study includes twelve patients (five left-sided and seven right-sided chest wall), who were planned and treated with CT-image based surface mould HDR brachytherapy for chest wall scar boost (CWB) using Catheter Flap Set ${ }^{\mathrm{TM}}$ (Varian Medical Systems, USA) that were given concurrently during external beam radiotherapy (EBRT) treatments. Since no guidelines are available for delineating clinical target volume (CTV) structure to be used for postmastectomy scar boost, the CTV in this study was a uniform 5-mm thick volume drawn at $5 \mathrm{~mm}$ beneath the skin (CTVhdr evl) and its extent was made conforming to the boost area marked on the skin and made visible in CT images by radiopaque wires. Results: Prescribed dose (PD) to CTVhdr_evl is $7.5 \mathrm{~Gy}$ in 3 fractions, and $2.5 \mathrm{~Gy}$ per fraction. The CTVhdr_evl volume receives the PD with mean $\mathrm{V}_{100 \%}, \mathrm{~V}_{98 \%}$ and $\mathrm{V}_{95 \%}$ values which are $98.57 \%, 99.63 \%$ and $100 \%$ respectively. The mean dose for heart (MHD) is 2.71 Gy in left-sided CWB and $1.80 \mathrm{~Gy}$ in right-sided CWB plans. Mean lung dose (MLD) is $2.48 \mathrm{~Gy}$ for ipsilateral lung and $0.76 \mathrm{~Gy}$ for contralateral lung. Maximum dose to contralateral breast is $4.93 \mathrm{~Gy}$ and the mean dose is $0.79 \mathrm{~Gy}$. The mean percent dose to the skin volume overlying the CTVhdr_evl is $138.6 \%$ and $3.7 \%$ of skin volume received $200 \%$ of the PD. Conclusion: The 3D image-based HDR surface mould achieved good CTV coverage with acceptable doses to OARs. Patient preparation, treatment planning, and execution in this method are less cumbersome and reproducible. Thus surface mould using flap applicator can be used whenever postmastectomy surgical scar boost is required.
\end{abstract}

Keywords: Postmastectomy Radiation; Surgical Scar Boost; HDR Surface Mould; Catheter Flap

\section{Introduction}

Postmastectomy radiotherapy (PMRT) to chest wall (CW) is recommended in locally advanced breast cancer. In the absence of radiotherapy (RT), loco-regional failure can occur in approximately $25 \%-40 \%$ of node-positive pa-

*Corresponding author. tients, and in up to $15 \%-20 \%$ of node-negative patients who do not receive systemic therapy [1]. Even after Danish and British Columbia trials, the locoregional risk reduction and impact on survival with PMRT are still debatable especially in patients with 1 to 3 positive nodes [2-8]. But, it is well established that the surgical scar in the $\mathrm{CW}$ is the most frequent site of locoregional recur- 
rence (LRR) [9,10].

Recent studies have suggested that when more than one of the adverse risk factors viz., young age, premenopausal status, tumour size, tumour grade, lymphovascular invasion, margin status, nodal ration, estrogen receptor status, tumour subtype, 21-gene recurrence score, and the genomic predictive index are present in the setting of nodal involvement, more aggressive locoregional management is warranted [11,12]. Even as the debate continues, the technological development in External Beam Radiotherapy (EBRT) like Intensity Modulated Radiotherapy (IMRT), Respiratory Gated Radiotherapy, and Volumetric Modulated Radiotherapy (VMAT), and Image-Guided Radiotherapy (IGRT), has made it now possible to deliver radiation to Planned Target Volumes (PTV) with minimal setup errors and with acceptable dose coverage, while sparing the organs at risk (OARs). Moreover, efforts are also made to develop atlas-based guidelines for implementing uniformity in delineation of the target and critical structures which are expected to minimize the interpersonal variations [13]. In PMRT, by implementing these technologies and using electronsphoton combinations, it is expected to bring down the pulmonary or cardiac toxicities [14-18].

As the scar in the chest wall is clearly the most common site of loco-regional failure, whenever there is a close or positive margin, and boosting the surgical scar area is considered with a dose of $10 \mathrm{~Gy}$ in 5 fractions. Usually enfacing electrons is used to deliver the boost dose. However, planning and preparation for electron treatment are complex, as electron cutouts and dose featherings require time and effort. In this study, an attempt is made to reduce this complexity by introducing HDR surface mould for the chest wall boost (CWB). While boost treatment during adjunct radiotherapy following breast conservation surgery is documented [11], not many data are available about effective methods for CWB during PMRT. A new method for CWB during PMRT using Catheter Flap $^{\text {TM }}$ (Varian Medical Systems, USA) surface mold HDR brachytherapy was introduced and its efficacy in dose coverage to tumour volume and sparing the underlying critical organs is evaluated.

\section{Methods and Materials}

\subsection{Patient Selection and Materials}

In this study, the inclusion criteria for surgical scar boost are women who have had mastectomy with positive or close surgical margins; and without cardiac or pulmonary co-morbidities. Twelve patients (five left-sided and seven right-sided chest wall), who had positive or close surgical margins were included prospectively in this study and planned for PMRT with sandwich scar boost using HDR flap surface mould. Commercially available CT/MR co- mpatible flexible Catheter Flap ${ }^{\circledR}$ (Varian Medical Systems, Palo Alto, CA, USA) with 20 channels was used in this study for HDR surface mould treatments (Figure 1). Each channel is separated by $1 \mathrm{~cm}$ and this flap is capable of treatments up to $200 \mathrm{~mm} \times 290 \mathrm{~mm}$ area. The catheters are slightly radiopaque and thus visible in the $\mathrm{CT}$ images. Contouring, segmentation and planning, evaluations are done by the same radiation oncologist and physicist to minimize interpersonal variations. Optimization and calculations were performed in BrachyVision ${ }^{\mathrm{TM}}$ (Version 10.0.42) which is integrated in the Eclipse treatment planning environment (Varian Medical Systems Inc., Palo Alto, CA, USA).

\subsection{Immobilization Methods and Plan Preparation}

Patients were immobilized with thermoplastic cast made on full carbon fiber breast board in the same supine position with the ipsilateral arm abducted above head as that required for EBRT for easy image registration. Surface markings were drawn around scar at a distance of $3 \mathrm{~cm}$ in the craniocaudal direction and $2-3 \mathrm{~cm}$ along the medial and lateral borders (Figure 2(a)) by the oncologist. Immobilization casts for EBRT were made separately. For CWB, the catheter flap was positioned beneath the cast and on the patient's chest wall so that the position of catheter flap is fixed in the same position throughout treatments and reproducible during subsequent fractions. Care was taken to keep the patients position same for both EBRT and HDR treatment plans for easy image registrations so that plan sums can be generated later for evaluation. Radioopaque wires were placed on the skin markings and CT scans were taken in the CT-simulator (Siemens Ltd., Germany) with $3 \mathrm{~mm}$ slice thickness separately for EBRT and for HDR. Registrations of the images were approved only after matching at least the treatment area (i.e., Chest Wall). Contouring for EBRT was done using RTOG atlas [13]. Before finalizing EBRT plan, HDR Brachytherapy planning was also done

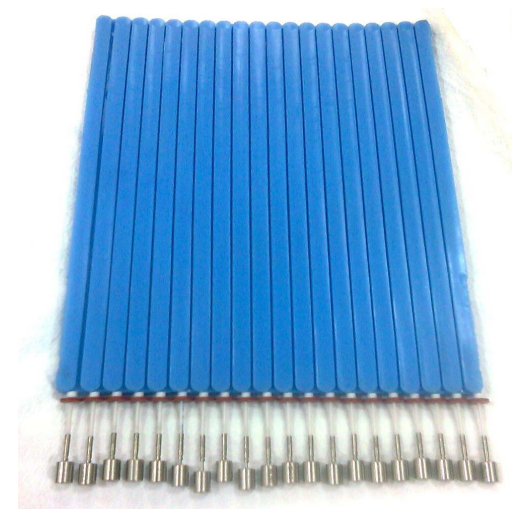

Figure 1. Catheter flap set ${ }^{\mathrm{TM}}$ (Varian medical systems, USA). 


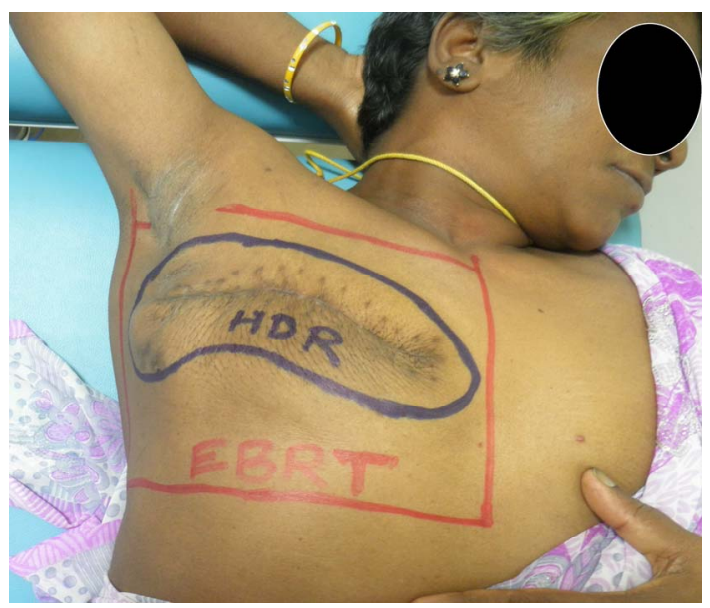

(a)

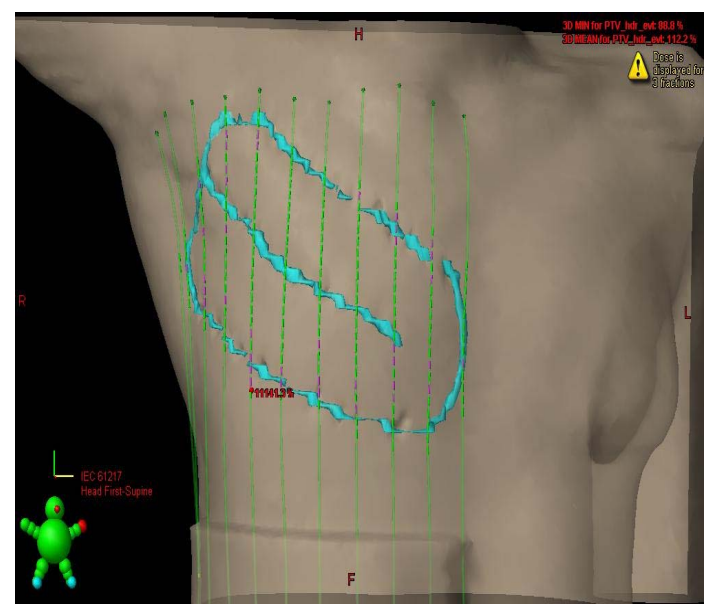

(c)

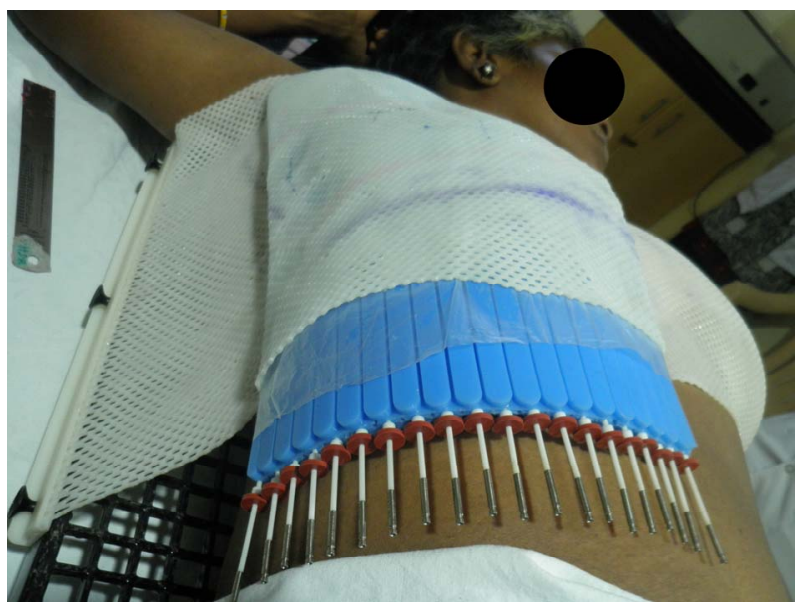

(b)

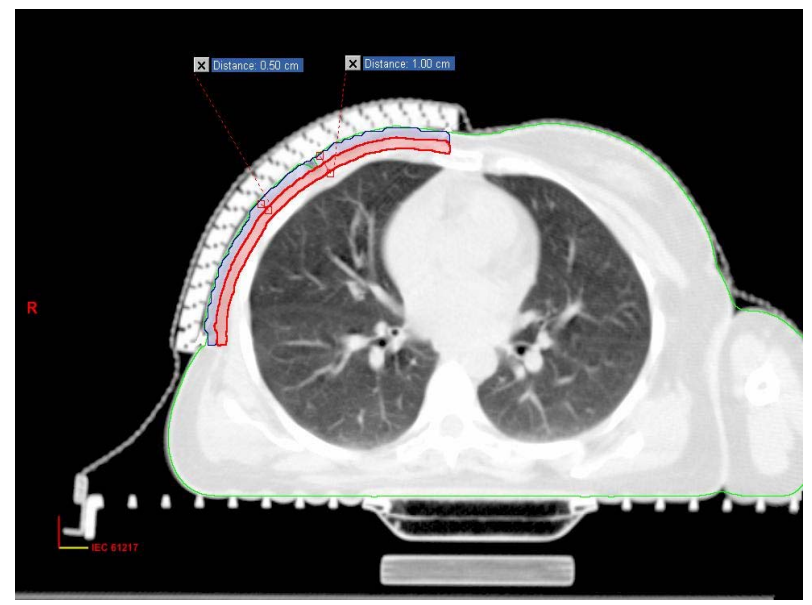

(d)

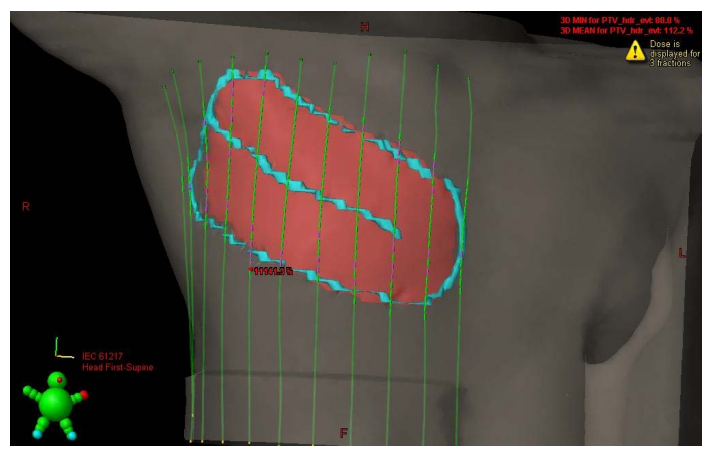

(e)

Figure 2. Method for CTV delineation: (a) Surface markings made on the patient; (b) Mould is prepared with immobilization and in the same position as in EBRT; (c) 3D view of the surface wire markings; (d) CTVhdr_evl (red) and overlying skin (blue) are delineated using wire images (other OAR structures are hidden); (e) 3D view of the reconstructed CTVhdr_evl (red) conforming to surface markings.

with the catheter flap as described below and got evaluated for both HDR plan and plan sum with EBRT.

\subsection{Method for CTV Delineation}

The CTV for HDR (CTVhdr_evl) is constructed using radio-opaque wire markers, which were placed along the scar and $3 \mathrm{~cm}$ in craniocaudal direction and $2-3 \mathrm{~cm}$ in lateral extension of the scar as shown in the Figure 2(a). Using the CT images a uniform CTVhdr_evl is constructed as a $5-\mathrm{mm}$ thick structure lying $5 \mathrm{~mm}$ beneath the skin surface (body) using extract wall \& cropping tools available in the contouring workspace of the plan- 
ning system and by using Boolean operators and 3D live view, the boundaries are clipped along the wire markers. (Figures 2(b)-(e)). Five-mm-thick skin volume structure is then created above the CTVhdr_evl structure up to the skin level for giving dose constraints during volume optimization of the HDR plan.

\subsection{Dose Prescription}

The dose prescription to CTV hdr_evl was 2.5 Gy per fraction given for 3 fractions so that the total dose to be delivered as scar boost is 7.5 Gy for all patients in this study. The EBRT dose prescription was 46 Gy in 23 fractions to entire ipsilateral chest wall and regional nodes with additional two fractions given to supraclavicular and axillary level 3 nodes. The CTVevl in EBRT is cropped to lie $5 \mathrm{~mm}$ from the skin level for better dose coverage in IMRT or VMAT planning. The HDR surface mould dose was limited to $7.5 \mathrm{~Gy}$ in 3 fractions which is less than the recommended $10 \mathrm{~Gy}$ in 5 fractions by ACR guidelines [11]. This was done because the surface mould usually delivers higher dose to the skin overlying the CTVhdr_evl $\left(\mathrm{D}_{90} \geq 120 \%\right)$ when compared to dose by enface electrons along with bolus. Thus giving 10 Gy with surface mould may result in higher skin reactions. The three HDR fractions are interdigitated with EBRT treatments and scheduled to be delivered after completion of every 5 fractions of EBRT, with no EBRT delivered on the days of HDR treatments.

\subsection{Catheter Reconstruction and Source Dwell Positionings}

The catheter auto-construction tool in the BrachyVi $\operatorname{sion}^{\mathrm{TM}}$ is used to reconstruct the catheters. Once the catheters are constructed, using the source dwell positions placement tool, sources with $5 \mathrm{~mm}$ step sizes were made to dwell only above this CTVhdr_evl area with two or three additional dwell positions beyond its boundary (Figure 2(d)). These procedures are completed usually in 20 to 30 minutes.

\subsection{Inverse Planning Optimization}

In this study treatment planning was done using inverse planning Adaptive Volume Optimization (AVOL) algorithm available in the BrachyVision ${ }^{\mathrm{TM}}$ Version 10.0.42. This optimization algorithm attempts to achieve the specified objectives and constraints with smoother dwell times and fewer hot spots inside the structures. The system default minimum and maximum dose limits to target are $95 \%$ and $120 \%$ with $100 \%$ priority for both, which were modified during later iterations so that $\mathrm{V}_{100 \%} \geq 98 \%$ is achieved. If dose to skin exceeds beyond $200 \%$, then the penalty score to skin constraint is set higher in subsequent iterations. As the planning optimization is interactive, normally two or three iterations are enough to achieve the desired dose distribution. But in a few patients studied here, after performing optimization, isodose reshaper tool was used to improve the dose distribution by dragging the isodose lines using mouse in selected CT slices to achieve required dose values in them. Suitable plans for evaluation are the ones whose $\mathrm{V}_{100 \%}$ is at least $95 \%$. Dose distributions are evaluated slice by slice qualitatively and then quantitatively by using dose volume histograms (DVHs).

\section{Results}

Various volume and dosimetric parameters that show the coverage of CTVhdr_evl are listed in Table 1. The statistical descriptive analysis (95\% CI of mean \& Quartile deviation) were done using GraphPad Prism 6 (v6.02) for Windows. $\mathrm{V}_{80 \%}$ and $\mathrm{V}_{90 \%}$ were chosen as these parameters indicate minimum dose received by the CTV. The parameters $\mathrm{V}_{95 \%}$ and $\mathrm{V}_{98 \%}$ show that there is a good coverage of dose to CTV $($ mean $=99.8 \%, \mathrm{SD}=0.47, \mathrm{n}=12$; and mean $=99.47 \%, \mathrm{SD}=0.93, \mathrm{n}=12$; respectively) and that the level of homogeneity of dose inside the CTV. $\mathrm{V}_{100 \%}(\%)$ indicates the proportion of CTV receiving at least the prescribed dose, and it is seen that $98.57 \%$ of the CTV volume receives $100 \%$ prescribed dose. To show the level of maximum dose inside the CTV, $\mathrm{V}_{150 \%}$ is chosen, as it is used routinely in reporting brachytherapy treatments, whose values (mean $=2.58 \%, \mathrm{SD}=2.38$, $\mathrm{n}=12$ ) in this study are acceptable.

Table 2 lists the dose-volume parameters for the heart. For the left-sided chest wall patients, the $\mathrm{D}_{\text {mean }}$, and $\mathrm{D}_{\text {median }}$ values are $36 \%$ and $33 \%$ of the prescribed dose respectively. The maximum dose to heart is around $71 \%$ of the prescribed dose. For the right-sided chest wall patients, the corresponding values are $24 \%, 23 \%$, and $50 \%$ respectively.

As for the other OARs, as given in Table 3, the dosevolume parameters were chosen as relevant to total dose of 7.5 Gy prescribed in this study. Thus, the volume parameter for lungs is limited to $\mathrm{V}_{5 \mathrm{~Gy}}$, apart from giving mean and maximum doses. For contralateral breast, $\mathrm{V}_{5 \%}$ and $\mathrm{V}_{10 \%}$ were given, which will be required if EBRT for the chest wall was delivered using IMRT or VMAT techniques, which invariably delivers low doses to this contralateral breast. Thus, the total value for $\mathrm{V}_{5 \%}$ and $\mathrm{V}_{10 \%}$, from plan sum of both EBRT and HDR, will give the input for calculating secondary cancer incidence probability.

Table 3 also lists the dose delivered to the skin. The parameters used are $\mathrm{V}_{150 \%}$ and $\mathrm{V}_{200 \%}$, as they give the level of high doses given to skin volumes. They also can be used to predict and to explain various skin reactions 
Table 1. CTV dose-volume parameters.

\begin{tabular}{|c|c|c|c|}
\hline CTV metrics & Mean (SD) n = 12 & Range & 95\% CI of mean \\
\hline & \multicolumn{3}{|c|}{ CTVhdr_evl volume: $78.79(21.11) \mathrm{cm}^{3}$} \\
\hline \multicolumn{4}{|c|}{ Volume Parameters } \\
\hline $\mathrm{V}_{80 \%}(\%)$ & $100(0.0)$ & $100-100$ & $100-100$ \\
\hline$V_{90 \%}(\%)$ & $99.95(0.14)$ & $99.5-100$ & $99.86-100$ \\
\hline$V_{95 \%}(\%)$ & $99.8(0.47)$ & $98.33-100$ & $99.80-100.1$ \\
\hline$V_{98 \%}(\%)$ & $99.47(0.93)$ & $96.61-100$ & $98.88-100.1$ \\
\hline $\mathrm{V}_{100 \%}(\%)$ & $98.57(1.44)$ & $94.49-99.95$ & $97.66-99.49$ \\
\hline $\mathrm{V}_{150 \%}(\%)$ & $2.58(2.38)$ & $0.29-7.88$ & $1.066-4.09$ \\
\hline \multicolumn{4}{|l|}{ Dose Parameters } \\
\hline$D_{90 \%}(\%)$ & $103.5(1.06)$ & $101.9-106.1$ & $102.8-104.2$ \\
\hline$D_{98 \%}(\%)$ & $100.7(1.79)$ & $95.82-102.9$ & $99.54-101.8$ \\
\hline
\end{tabular}

Abbreviations: $\mathrm{SD}=$ standard deviation; $\mathrm{V}_{\mathrm{x} \%}(\%)=$ percent volume receiving at least $\mathrm{x} \%$ of the prescribed dose; $\mathrm{D}_{\mathrm{y} \%}(\%)=$ percent dose received by the $\mathrm{y} \%$ of the volume.

Table 2. Dose volume parameters for heart.

\begin{tabular}{|c|c|c|c|c|c|c|}
\hline \multirow{2}{*}{ Metric } & \multicolumn{3}{|c|}{ Left side chest wall $(n=5)$} & \multicolumn{3}{|c|}{ Right side chest wall $(n=7)$} \\
\hline & Mean (SD) & Range & 95\% CI of Mean & Mean (SD) & Range & 95\% CI of Mean \\
\hline \multicolumn{7}{|l|}{ Heart } \\
\hline Volume in cc & \multicolumn{3}{|c|}{$396(116.6)$} & \multicolumn{3}{|c|}{$419.5(105.1)$} \\
\hline$D_{\text {mean }}$ (cGy) & $271.6(42.87)$ & $231-335.7$ & $218.4-324.8$ & $180.1(27.13)$ & $149.2-220.6$ & $155-205.2$ \\
\hline $\mathbf{D}_{\text {median }}$ (cGy) & $248.2(45.23)$ & $203.9-21.5$ & $192.1-304.4$ & $172.7(30.55)$ & $133.4-216.7$ & $144.4-200.9$ \\
\hline$D_{\max }(\mathrm{cGy})$ & $532.5(88.22)$ & $420.5-658.8$ & $422.9-642$ & $374(48.13)$ & $313.4-447.5$ & $329.5-418.5$ \\
\hline$V_{5 G y}(\%)$ & $4.886(4.562)$ & $0-10.28$ & $-0.78-10.55$ & 0 & & \\
\hline
\end{tabular}

Abbreviations: $\mathrm{SD}=$ standard deviation; $\mathrm{CI}=$ confidence interval; $\mathrm{D}_{2 \%}(\mathrm{cGy})=$ Dose received by $2 \%$ of the volume; $\mathrm{V}_{5 \mathrm{~Gy}}(\%)=$ percent volume receiving at least 5 Gy dose.

Table 3. Dose volume parameters for OARs.

\begin{tabular}{|c|c|c|c|}
\hline \multirow{2}{*}{ Metrics } & \multicolumn{3}{|c|}{ Both right \& left side chest wall $(n=12)$} \\
\hline & Mean (SD) & Range & 95\% CI of Mean \\
\hline \multicolumn{4}{|c|}{ Ipsilateral Lung } \\
\hline Volume in $\mathrm{cm}^{3}$ & & $876.6(294.2)$ & \\
\hline $\mathbf{D}_{\text {Mean }}(\mathrm{cGy})$ & $248.2(28.23)$ & $201.4-282.3$ & $230.3-266.2$ \\
\hline$D_{\text {Max }}$ (cGy) & $711.6(105.8)$ & $438.4-846.6$ & $644.4-778.9$ \\
\hline $\mathrm{D}_{2 \%}$ (cGy) & $602.2(54.69)$ & $491.5-692.1$ & $567.5-637$ \\
\hline$V_{5 G y}(\%)$ & $3.606(4.327)$ & $0-12.63$ & $0.86-6.35$ \\
\hline \multicolumn{4}{|c|}{ Contralateral Lung } \\
\hline Volume in $\mathbf{c m}^{3}$ & & $802.9(188.2)$ & \\
\hline$D_{\text {Mean }}(c G y)$ & $76.13(15.58)$ & $59.2-113.8$ & $66.23-86.03$ \\
\hline$D_{\text {Max }}$ (cGy) & $305.6(126.9)$ & $148.2-557.4$ & $224.9-386.2$ \\
\hline $\mathrm{V}_{5 \mathrm{~Gy}}(\%)$ & $0(0)$ & $0-0$ & \\
\hline \multicolumn{4}{|c|}{ Contralateral Breast } \\
\hline Volume in $\mathrm{cm}^{3}$ & & $662.1(263.1)$ & \\
\hline $\mathbf{D}_{\text {Mean }}(\mathrm{cGy})$ & $79.28(14.02)$ & $55.2-102.5$ & $70.38-88.19$ \\
\hline$D_{\text {Max }}$ (cGy) & $492.7(166.2)$ & $273.9-765.4$ & $387.1-598.2$ \\
\hline $\mathrm{V}_{5 \mathrm{~Gy}}(\%)$ & $0.27(0)$ & $0.27-0.27$ & \\
\hline$V_{5 \%}(\%)$ & $89.22(11.44)$ & $62.45-100$ & $81.95-96.48$ \\
\hline$V_{10 \%}(\%)$ & $34.69(9.659)$ & $17.49-53.1$ & $28.55-40.82$ \\
\hline \multicolumn{4}{|c|}{ Skin } \\
\hline Volume in $\mathrm{cm}^{3}$ & & 78.43 (22.67) & \\
\hline$D_{\text {Mean }}(\%)$ & $138.6(3.183)$ & $131.7-143.8$ & $136.5-140.6$ \\
\hline$V_{150 \%}(\%)$ & $18.85(3.897)$ & $10.93-25.7$ & $16.38-21.33$ \\
\hline$V_{200 \%}(\%)$ & $3.686(1.765)$ & $1-7.49$ & $2.565-4.807$ \\
\hline
\end{tabular}

Abbreviations: $\mathrm{SD}=$ standard deviation; $\mathrm{CI}=$ confidence interval; $\mathrm{D}_{\text {Mean }}, \mathrm{D}_{\mathrm{Max}}=$ Mean, Maximum doses; $\mathrm{D}_{2} \%(\mathrm{cGy})=\mathrm{Dose}$ received by $2 \%$ of the volume; $\mathrm{V}_{5 \mathrm{~Gy}}(\%)=$ percent volume receiving at least $5 \mathrm{~Gy}$ dose; $\mathrm{V}_{\mathrm{x} \%}(\%)=$ percent volume receiving at least $\mathrm{x} \%$ of the dose. 
during and post radiotherapy.

\section{Discussion}

\subsection{CTV Coverage}

It can be seen that the CTVhdr_evl has well received the prescribed dose as the mean values for $\mathrm{V}_{95 \%}, \mathrm{~V}_{98 \%}$, and $\mathrm{V}_{100 \%}$ were $99.8 \%(\mathrm{SD}=0.47), 99.47 \%(\mathrm{SD}=0.93)$, and $98.57 \%(\mathrm{SD}=1.47)$ respectively and their minimum doses respectively were $98.33 \%, 96.61 \%$, and $94.49 \%$ (Table 1). The dose coverage to $90 \%$ and $98 \%$ of the CTV volume was also well covered as indicated by $\mathrm{D}_{90 \%}$ and $\mathrm{D}_{98 \%}$ respectively with their mean values of $103.5 \%$ $(\mathrm{SD}=1.06)$ and $100.7 \%(\mathrm{SD}=1.79)$, with the minimum value $95.8 \%$

The graph in Figure 3 shows that there is high degree of uniformity of the dose in the CTV, as the interquartile ranges (IQRs) lie within $99 \%$ to $100 \%$ for $\mathrm{V}_{90 \%}, \mathrm{~V}_{95 \%}$, and $\mathrm{V}_{98 \%}$. The IQR for $\mathrm{V}_{100 \%}$ is $99.6 \%-98.3 \%$.

Hence, the CTVhdr_evl receives the full prescribed dose uniformly.

\subsection{Dose to Heart}

The mean and maximum dose (SD) to heart in left-sided chest wall patients were $2.7 \mathrm{~Gy}(0.42)$, and $5.3 \mathrm{~Gy}(0.88)$ respectively, which were $36 \%$ and $71 \%$ of the PD. The mean dose is similar to the reported heart dose delivered (2.3 Gy (0.7)) for 50 patients treated but when delivered with 40 Gy in 15 fractions to left-sided breast cancers [19]. These results are also comparable with mean cardiac doses reported ( $\mathrm{D}_{\text {mean }}$ of 2.45 for the heart and 3.29 Gy for the ventricles) when using balloon-based HDR brachytherapy [20]. The volume receiving $5 \mathrm{~Gy}$ is $4.89 \%$ in patient requiring left-side chest wall scar boost (Figure 4).

\subsection{Dose to Lungs}

The mean ipsilateral lung dose (MLD) is $2.48 \mathrm{~Gy}$ (SD = 0.28 , range 2.01 to $2.82 \mathrm{~Gy}$ ) (Figure 5). $\mathrm{D}_{2 \%}$ and $\mathrm{D}_{\max }$

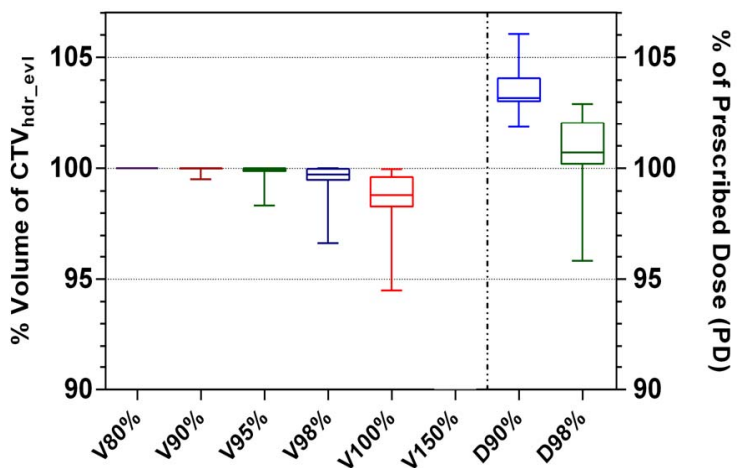

Figure 3. Box-and-whisper plot showing the coverage of CTV.
Dose to Heart

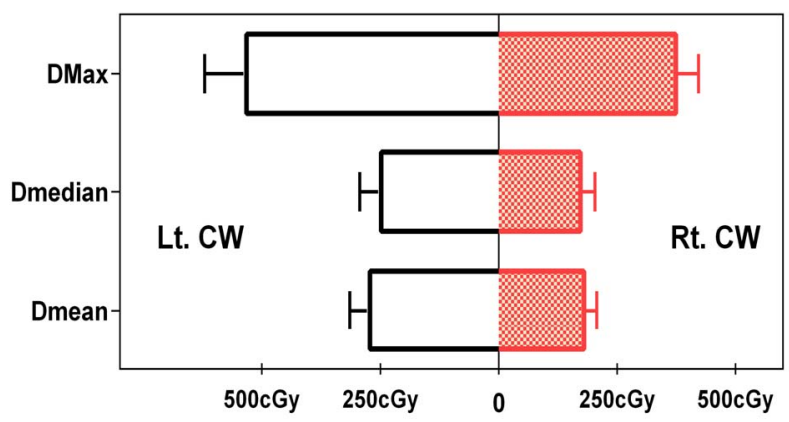

Figure 4. Dose to heart.

Dose to Lungs

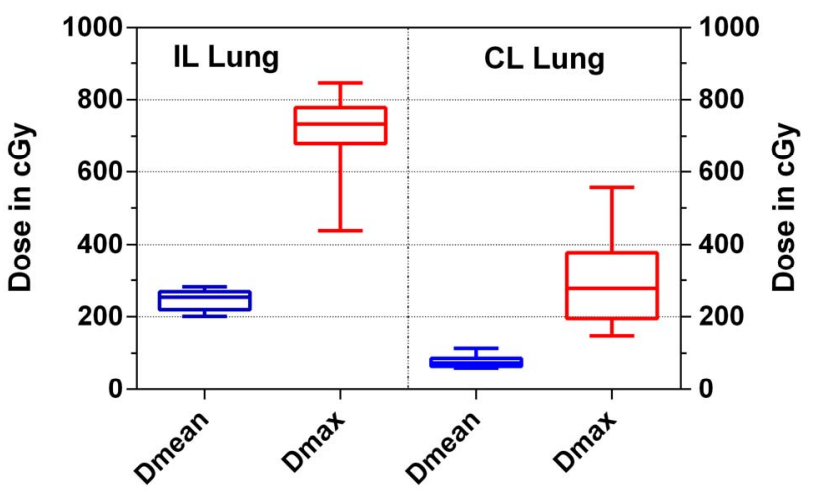

Figure 5. Dose to lungs (IL = ipsilateral, $\mathrm{CL}=$ contralateral).

values are $6.02 \mathrm{~Gy}(\mathrm{SD}=0.54$, range 4.91 to 6.92$)$ and $7.11 \mathrm{~Gy}(\mathrm{SD}=1.05$, range 4.38 to 8.47 ), which are about $80 \%$ and $95 \%$ of the PD. The volume of the lung receiving 5 Gy is $6.6 \%$. These values are well within the lung tolerance dose (V30Gy $<20 \%$ \& V20Gy $<30 \%-35 \%$, with MLD of $<10$ Gy when summed with EBRT dose using IMRT/VMAT plans.

The mean contralateral lung dose (MLD) is 0.76 Gy ( $\mathrm{SD}=0.16$, range 0.59 to $1.14 \mathrm{~Gy}$ ). The mean $\mathrm{D}_{\max }$ value is $3.05 \mathrm{~Gy}$ ( $\mathrm{SD}=1.27$, range 1.48 to $5.57 \mathrm{~Gy})$ which are about $41 \%$ of the PD. The volume of the contralateral lung receiving $5 \mathrm{~Gy}$ is negligible (zero in $\mathrm{DVH}$ ).

\subsection{Dose to Contralateral Breast}

The maximum dose to contralateral breast is $4.92 \mathrm{~Gy}$ ( $\mathrm{SD}=1.66$, range 2.74 to $7.65 \mathrm{~Gy}$ ), which is $65 \%$ of the $\mathrm{PD}$ and $\mathrm{D}_{\text {mean }}$ is 0.8 Gy $(\mathrm{SD}=0.14$, range 0.55 to 1.03 Gy) (Figure 6). The $\mathrm{V}_{5 \%}$ and $\mathrm{V}_{10 \%}$ values are $89 \%(\mathrm{SD}=$ 0.11 , range $62 \%$ to $100 \%$ ) and $35 \%(\mathrm{SD}=9.7$, range $17 \%$ to $53 \%$ ) respectively. Thus around $90 \%$ of the volume is exposed to low doses. However, the dose due to HDR is significantly less compared to scattered from tangential fields by EBRT [19]. 


\subsection{Dose to Skin}

The mean dose to skin is $138.6 \%$ ( $\mathrm{SD}=3.18$, range $131.7 \%$ to $143.8 \%$ ) of the $\mathrm{PD}$, and the higher doses, like $150 \%$ and $200 \%$ doses were received by $18.85 \%$ (SD $=$ 3.89 , range $10.93 \%$ to $25.7 \%$ ), and $3.686 \%(\mathrm{SD}=1.76$, range $1 \%$ to $7.49 \%$ ) of skin volume (Figure 7 ). This is in expected lines as the skin lies between the source and the CTVhdr_evl. Moreover this is the reason for limiting the scar boost dose to 7.5 Gy in 3 fractions, although dose recommended is $10 \mathrm{~Gy}$ in 5 fractions [11]. Higher skin dose is desirable during scar boost and it is the standard feature in surface mould brachytherapy.

\section{Conclusions}

An alternative method for postmastectomy surgical scar boost is designed here, which does not have the complexities of the electron beam and electron arc techniques. The latter techniques involve the design of custom made cutouts to collimate the electron beam to the area of irradiation, since the chest wall is usually curvy and also contains lung, bone and soft tissue heterogeneities, posing a heavy challenge to the planner. These challenges, notwithstanding there are electrons, are still preferred over EBRT photons due to their favourable depth dose characteristics and better OAR sparing ability.

This HDR surface mould technique is simpler and requires only an additional immobilization cast to be made

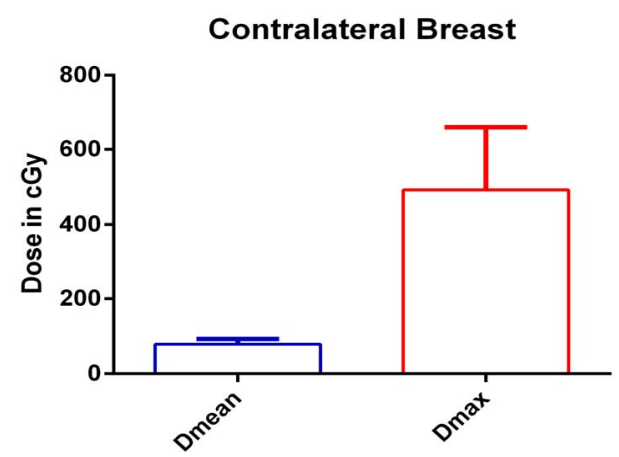

Figure 6. Dose to contralateral breast.

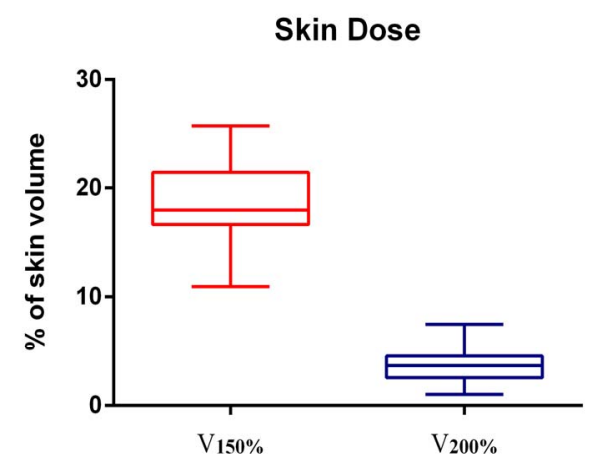

Figure 7. Dose to skin overlying the CTV. ensuring reproducibility over fractions. The use of immobilization cast also minimizes air gaps between the flap and skin. If the scar length is more than $20 \mathrm{~cm}$, then this catheter flap orientation has to be changed so that the treatment area is covered by the flap. The brachytherapy volume-based inverse planning is interactive, and DVHbased constraints can be given as input and do not take as much time as that of inverse planning of EBRT. Planner can still adjust the dose distribution, if needed, when optimization is not satisfactory.

Finally, HDR brachytherapy fractions can be interdigitated with EBRT, reducing the overall treatment time. It also reduces the Linac's usage time.

The downside of this HDR surface mould is that it invariably delivers low doses to lungs and contralateral breast. While the dose to lungs cannot be reduced, the low doses to the contralateral breast can be minimized by shielding it using lead sheets.

Thus HDR surface mould is a promising technique and can be made as a routine choice in the clinic, whenever surgical scar boost is planned during PMRT.

\section{REFERENCES}

[1] R. Arriagada, L. E. Rutqvist and M. G. Le, "Postmastectomy Radiotherapy: Randomized Trials," Seminars in Radiation Oncology, Vol. 9, No. 3, 1999, pp. 275-286.

[2] T. A. Buchholz, E. A. Strom, G. H. Perkins and M. D. McNeese, "Controversies Regarding the Use of Radiation after Mastectomy in Breast Cancer," The Oncologist, Vol. 7, No. 6, 2002, pp. 539-546. http://dx.doi.org/10.1634/theoncologist.7-6-539

[3] L. B. Marks, J. Zeng and L. R. Prosnitz, "One to Three versus Four or More Positive Nodes and postmastectomy Radiotherapy. Time to End the Debate," Journal of Clinical Oncology, Vol. 26, No. 13, 2008, pp. 2075-2077. http://dx.doi.org/10.1200/JCO.2007.15.5200

[4] N. S. Russell, I. H. Kunkler, G. V. Tienhoven, P. A. Canney, J. Thomas, J. Bartlett, et al., "Postmastectomy Radiotherapy: Will the Selective Use of Postmastectomy Radiotherapy Study End the Debate?" Journal of Clinical Oncology, Vol. 27, No. 6, 2009, pp. 996-1001. http://dx.doi.org/10.1200/JCO.2008.18.7062

[5] M. Overgaard, P. S. Hansen, J. Overgaard, C. Rose, M. Andersson and F. Bach, et al., "Postoperative Radiotherapy in High-Risk Premenopausal Women with Breast Cancer Who Receive Adjuvant Chemotherapy. Danish Breast Cancer Cooperative Group 82b Trial," The New England Journal of Medicine, Vol. 337, No. 14, 1997, pp. 949-955. http://dx.doi.org/10.1056/NEJM199710023371401

[6] M. Overgaard, M. B. Jensen, J. Overgaard, P. S. Hensen, C. Rose, M. Andersson, et al., "Postoperative Radiotherapy in High-Risk Postmenopausal Breast-Cancer Patients Given Adjuvant Tamoxifen: Danish Breast Cancer Cooperative Group 82c Randomized Trial," Lancet, Vol. 353, No. 9165, 1999, pp. 1641-1648. 
http://dx.doi.org/10.1016/S0140-6736(98)09201-0

[7] M. Overgaard, H. M. Neilsoen and J. Overgaard, "Is the Benefit of Postmastectomy Irradiation Limited to Patients with Four or More Positive Nodes, as Recommended in International Consensus Reports? A Subgroup Analysis of the DBCG 82 b\&c Randomized Trials," Radiotherapy \& Oncology, Vol. 82, No. 3, 2007, pp. 247-253. http://dx.doi.org/10.1016/j.radonc.2007.02.001

[8] J. Ragaz, I. A. Olivotto, J. J. Spinelli, N. Phillips, S. M. Jackshon, K. S. Wilson, et al., "Locoregional Radiation Therapy in Patients with High-Risk Breast Cancer Receiving Adjuvant Chemotherapy: 20-Year Results of the British Columbia Randomized Trial," Journal of the National Cancer Institute, Vol. 97, No. 2, 2005, pp. 116-126. http://dx.doi.org/10.1093/jnci/djh297

[9] H. M. Nielson, M. Overgaard, C. Grau, A. R. Jenson and J. Overgaard, "Study of Failure Pattern among High-Risk Breast Cancer Patients with or without Postmastectomy Radiotherapy in Addition to Adjuvant Systemic Therapy: Long-Term Results from the Danish Breast Cancer Cooperative Group DBCG 82 b and c Randomized Studies," Journal of Clinical Oncology, Vol. 24, No. 15, 2006, pp. 2268-2275. http://dx.doi.org/10.1200/JCO.2005.02.8738

[10] A. Recht, R. Gray, N. E. Davidson, B. L. Fowble, L. J. Solin, F. J. Cummins, et al., "Locoregional Failure 10 Years after Mastectectomy and Adjuvant Chemotherapy with or without Tamoxifen without Irradiation: Experience of the Eastern Cooperative Oncology Group," Journal of Clinical Oncology, Vol. 17, No. 6, 1999, pp. 16891700 .

[11] M. E. Taylor, B. G. Haffty, R. Rabinovitch, D. W. Arthur, F. E. Halberg, E. A. Strom, et al., "ACR Appropriateness Criteria $^{\circledR}$ on Postmastectomy Radiotherapy," International Journal of Radiation Oncology*Biology*Physics, Vol. 73, No. 4, 2009, pp. 997-1002. http://dx.doi.org/10.1016/j.ijrobp.2008.10.080

[12] S. M. Macdonald, R. F. Abi-Raad, M. A. Alm El-Din, D. W. Arthur, F. E. Halberg, et al., "Chest Wall Radiotherapy; Middle Ground for Treatment of Patients with One to Three Positive Lymph Nodes after Mastectomy," International Journal of Radiation Oncology*Biology* Physics, Vol. 75, No. 5, 2009, pp. 1297-1303. http://dx.doi.org/10.1016/j.ijrobp.2008.10.080

[13] Radiation Therapy Oncology Group, "Breast Cancer Atlas for Radiation Therapy Planning: Consensus Defini- tions," 2013.

http://www.rtog.org/CoreLab/ContouringAtlases/BreastC ancerAtlas.aspx

[14] E. Gez, N. Ashaf, E. R. Bar-Deroma, E. Rosenblatt and A. Kuten, "Postmastectomy Electron-Beam Chest-Wall Irradiation in Women with Breast Cancer," International Journal of Radiation Oncology*Biology*Physics, Vol. 60, No. 4, 2004, pp. 1190-1194. http://dx.doi.org/10.1016/j.ijrobp.2004.05.036

[15] H. K. Hamdy and M. S. Zikry, "Post Mastectomy Chest Wall Irradiation Using Mixed Electron-Photon Beams with or without Isocentric Technique," Gulf Journal of Oncology, No. 3, 2008, pp. 33-40.

[16] C. W. Hurkmans, B. C. J. Cho, E. Damen, L. Zijp and B. J. Mijnheer, "Reduction of Cardiac and Lung Complication Probabilities after Breast Irradiation Using Conformal Radiotherapy with or without Intensity Modulation," Radiotherapy \& Oncology, Vol. 62, No. 2, 2002, pp. 163171. http://dx.doi.org/10.1016/S0167-8140(01)00473-X

[17] M. S. Moran and B. G. Haffty, "Radiation Techniques and Toxicities for Locally Advanced Breast Cancer," Seminars in Radiation Oncology, Vol. 19, No. 4, 2009, pp. 244-255. http://dx.doi.org/10.1016/j.semradonc.2009.05.007

[18] S. M. Macdonald, R. Jimenez, P. Paetzold, J. Adams, J. Beatty and T. F. DeLaney, et al., "Proton Radiotherapy for Chest Wall and Regional Lymphatic Radiation; Dose Comparisons and Treatment Delivery," Radiation Oncology, Vol. 24, No. 8, 2013, pp. 71-77. http://dx.doi.org/10.1186/1748-717X-8-71

[19] C. W. Taylor, J. M. Povall, P. McGale, A. Nisbet, D. Dodwell, J. T. Smith, et al., "Cardiac Dose from Tangential Breast Cancer RT in the Year 2006," International Journal of Radiation Oncology*Biology*Physics, Vol. 72, No. 2, 2008, pp. 501-507. http://dx.doi.org/10.1016/j.ijrobp.2007.12.058

[20] V. Valakh, Y. Kim, E. D. Werts and M. G. Trombetta, “A Comprehensive Analysis of Cardiac Dose in Balloon-Based High-Dose-Rate Brachytherapy for Left-Sided Breast Cancer," International Journal of Radiation Oncology* Biology*Physics, Vol. 82, No. 5, 2012, pp. 1698-1705. http://dx.doi.org/10.1016/j.ijrobp.2011.02.058 